\title{
Challenges in Grid Integration of Renewable Energy Systems via Inverter: A Survey
}

\author{
Sourabh Kedar \\ M. Tech Scholar \\ RKDF Institute of Science and Technology \\ SRK University \\ Bhopal, India \\ sourabhkedar194@gmail.com
}

\author{
Mr. Santosh Singh Negi \\ Assistant Professor \\ RKDF Institute of Science and Technology \\ SRK University \\ Bhopal, India \\ santoshsnegi@gmail.com
}

\begin{abstract}
Solar photovoltaic (PV) systems have mainly been used in the past decade. Inverter-powered photovoltaic grid topologies are widely used to meet electricity demand and to integrate forms of renewable energy into power grids. Meeting the growing demand for electricity is a major challenge today. This paper provides a detailed overview of the topological trend of inverters with connection to the photovoltaic grid, as well as the advantages, disadvantages and main characteristics of the individual inverters. For proper integration into a network, coordination between the supporting devices used for reactive power compensation and their optimal reactive power capacity for grid current stability is important.
\end{abstract}

Keywords: inverter topologies; grid; inverter; PV; reactive power.

\section{INTRODUCTION}

Grid-connected photovoltaic systems are grid-connected power generation systems. The generated solar photovoltaic energy must be processed using a grid-connected inverter before it can be put into operation. This inverter is located between the photovoltaic solar generator and the power grid. It can be a single unit or a set of small inverters connected to each PV unit. Due to the reduction of power electronics costs and the advancement of renewable energy technology, there is a strong incentive for the energy sector to use solar photovoltaic energy and connect it to a medium or medium low voltage distribution network. .

This solar photovoltaic has dominated all other forms of electricity generation. With this growing trend in the use of photovoltaic solar energy, it becomes even more important to look at the obstacles encountered when extracting energy from solar photovoltaic systems and then exporting or integrating it into the grid. The most important factors to consider when integrating photovoltaic solar energy into the grid are:

1. Cost reduction during the electrical conversion phase

2. Improved reliability of the converter used

3. Harmonics reduction in the received output current

4. Reduced number of switches / components used for network integration

5. Ensure continuity of supply by providing backup power to the PVs.

6. Control of active and reactive power

7. Maintaining a constant DC intermediate circuit voltage by means of an appropriate circuit diagram

8. Find the maximum power point of a PV module using maximum power point detection techniques.

\section{LITERATURE REVIEW}

Ooi H. P. Gabriel et al. [1] Multi-megawatt photovoltaic (PV) frameworks are generally utilized in power frameworks because of the extreme drop in PV module costs. Multi-string structure is considered for elite PV frameworks because of the increment in general force transformation effectiveness and measured quality contrasted with incorporated geography. High force transformation can be effectively accomplished by equal interfacing various matrix associated two-stage voltage source inverters (VSI 2L). Be that as it may, this design convolutes control projects. So this issue can be settled by supplanting these $2 \mathrm{~L}$ VSIs with a solitary high force three phase impartial point inverter (3L-NPC). Moreover, PNJ 3L acquires a few intriguing benefits, for example, Better force quality with 
diminished complete consonant bending (THD), higher generally effectiveness and decreased expenses. What's more, voltage-situated control with relative reverberation (VOC-PR) joined with vector versatile spatial balance (ASVM) is offered for enormous scope PV frameworks with network associated NPC inverters.

J. Pou et al. [2] this article inspects the Low Voltage Ride through (LVRT) ability of the HB full line inverter in a Grid Connected Photovoltaic Power Plant (GCPVPP). A control calculation is executed for the secluded DC/DC converter, which makes up for capacitor voltages during voltage drops and decreases the second consonant voltage wave of the halfway circuit capacitors. Matrix associated full-column HB inverter execution with LVRT ability is analyzed on a solitary line, three-stage CHB 3 MVA geography for GCPVPP associated with a $6.6 \mathrm{kV}$ network. In each stage, no single strings per stage, however all PV strings are associated with all stages through disengaged DC/DC converters. The issue of the force lopsidedness per stage and the second symphonious voltage of the middle of the road circuit capacitors is decreased by utilizing this geography.

A. Camacho et al. [3] this article suggests that responsive force control directs the most extreme and least stage voltages at the basic coupling point inside the cutoff points indicated in the lattice codes for ceaseless activity. In case of even three-stage voltage drops, the control builds the voltage in each stage over the directed lower limit giving the receptive force of the positive grouping.

In case of topsy-turvy voltage drops, the receptive forces are joined with a positive and negative succession to deftly increment and equilibrium the stage voltages. The most extreme stage voltage is changed underneath as far as possible and the base stage voltage is changed simply over as far as possible. The proposed control methodology is tried considering a distant organization disappointment and a high organization impedance. Chosen test results are introduced to approve the conduct of the control plot.

JundiJia et al. [4] In this article, the current VSC control techniques in the event of lopsided blames for hamper are examined in two gatherings, specifically a force trademark arranged control procedure and a voltage support situated control system. Since the deficiency current provided by the converters should be restricted inside safe working cutoff points, considering the limits of the semiconductors, the issue of restricting the converter current is likewise examined.
Converter-based age can contrast altogether from ordinary alternators in case of a framework disappointment.

To assess the potential impacts of future converter-put together force frameworks with respect to insurance transfers, different voltage source converter (VSC) current control systems ought to be considered in case of deviated issues, since the exhibition of converters essentially rely upon their control destinations.

\section{IMPACTS Of CONNECTING PV System TO THE GRID}

If the PV penetration is really high Photovoltaic systems can subject the grid to several negative impacts. They are

i) Reverse power flow,

ii) Overvoltage along Distribution feeders,

iii) Voltage control difficulty,

iv) Phase unbalance,

v) Power Quality problems,

vi) Increased Reactive power and

vii) Islanding detection difficulty.

It considers the following three impacts.

1] Power quality problems/Harmonics

The inverter is the core of the photovoltaic framework associated with the network and is answerable for the nature of the energy took care of into the lattice. On account of nonstraight loads, inverters likewise bring music into the framework when changing direct current over to rotating current. Symphonious flows cause voltage drop and mutilation of the stock voltage. Sounds can likewise cause reverberation in the force framework, which can cause glitches, abbreviate life, or forever harm electrical hardware. [3].

\section{2] Increased Reactive}

PV power inverters typically work with a force factor of one. Little mortgage holders who take part in a motivator program are charged dependent on their kilowatt-hour yield as opposed to their hourly kilovolt-ampere yield.

They along these lines like to utilize photovoltaic inverters with power consider 1 request to expand the creation of dynamic energy and, in this manner, their profit from speculation. Subsequently, the receptive force necessity of the photovoltaic framework is negligible. Along these lines, the organization is answerable for giving the greater part of the receptive force and permits the conveyance transformer to work with a low force factor.

\section{3] Islanding Detection}


The condition in which the nearby planetary group keeps on providing capacity to the heap even without network power is called an island. Segregation can be hazardous for utility laborers who may not know that a circuit is as yet dynamic during fix or support.

The sun powered inverter should consequently perceive the island protection and separate the photovoltaic framework in case of a network disappointment.

This capacity of the photovoltaic framework is designated "hostile to islanding". These impacts rely upon the size and area of the photovoltaic framework. As per the Solar America Board of Codes and Standards (Solar ABCs), photovoltaic frameworks are separated into three classes dependent on framework orders.

\section{SYSTEM CONFIGURATION AND CONTROL}

In a conventional framework associated framework, the force transformation unit is associated straightforwardly to the network with no heap. The framework arrangement of a solitary stage DG working in network associated mode. The framework comprises of a DC power source, a voltage source inverter (VSI), a yield LC channel, a nearby burden and a public matrix

The reason for the DG framework is to give power to its nearby burden as well as taking care of into the lattice/transferring abundance capacity to the matrix at the regular coupling point (PCC). To create great force, the force that DG communicates to the matrix should be adjusted, sinusoidal and with a low THD. Because of the mutilation of the line voltage and the neighborhood non-direct burden present on the force matrix.

\section{DFIG CONFIGURATION}

The generator is designed utilizing an injury rotor acceptance generator (WRIG) with the stator windings associated straightforwardly to the mains and the rotor windings associated with the stator terminal by means of the AC/DC/AC converter. A transformer is utilized to control the voltage level between the matrix and the network side converter. The plan of the DFIG design is appeared in this figure. 1.

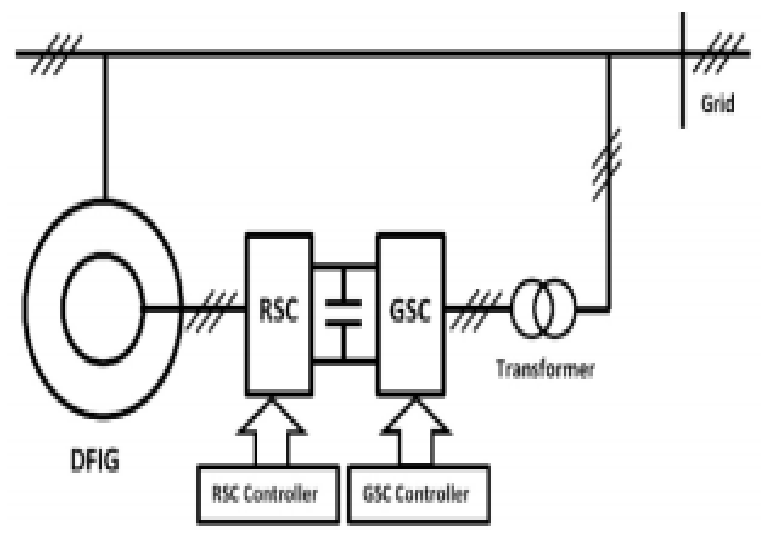

Fig. 1 DFIG Configuration

The consecutive converter is a bidirectional converter comprising of two conventional voltage source converters with heartbeat width tweak (PWM) and a typical DC transport with a middle of the road circuit capacitor.

Consecutive converters are regularly utilized in DFIG-based frameworks for wind applications to create more force. Contingent upon the decoupled control system and intricacy, DFIG can be utilized in both the sub-coordinated speed range and the super-simultaneous speed range. This kind of change framework comprises of two PWM converters with a halfway circuit capacitor. The GSC ought to be controlled so the middle of the road circuit voltage stays steady and is additionally answerable for controlling the receptive force of the DFIG and the organization.

The control technique of the rotor side converter (RSC) fundamentally considers the control of the electromagnetic force and the excitation flows of the rotor. The whole control procedure is characterized in a coordinated reference framework isolated into $\mathrm{d}-\mathrm{q}$ parts.

\section{INVERTER TOPOLOGY}

In the event that the produced power is transferred to the framework or utilized by AC loads, at that point DC-DC converters (inverters) should be utilized. Inverters can be single-stage or three-stage. There are four regular framework coordinated inverters for photovoltaic frameworks: incorporated inverter framework, string inverter framework, multi-string inverter framework, and miniature lattice inverter framework (AC modules).

The focal inverter is the past innovation and depends on focal inverters which have an enormous number of PV modules 
associated with the lattice. The PV modules are associated in arrangement (called a chain). These strings are associated in corresponding with string diodes to accomplish high force levels. String inverters are the current innovation and are the little sort of focal inverter that each string associates with the inverter.

Multi-string inverters have numerous strings and are associated with a typical DC-AC inverter with its own DC-DC converter. String inverters are superior to focal framework inverters because of their individual controllability. The square chart of a three-stage DC-AC inverter associated with the network is appeared in Fig. 2.

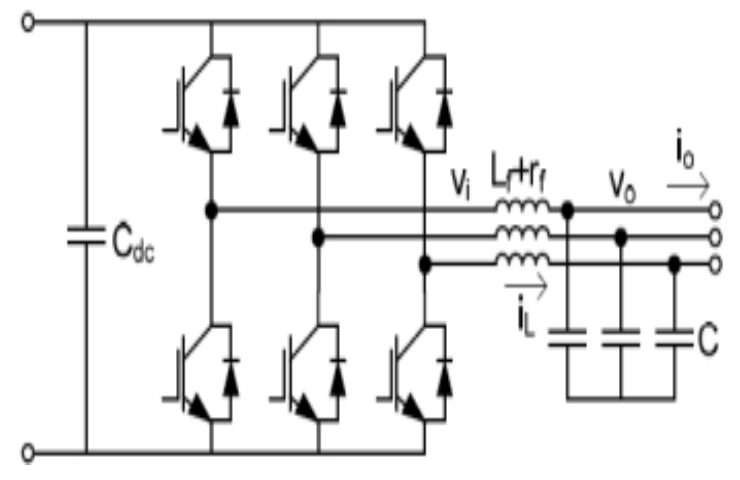

Fig. 2 Block diagram of three phase inverter

VII. CONCLUSION

Photovoltaic systems have become a mature technology for generating electricity. However, they introduce many negative effects into power grids. Studies on three of these effects were presented. Reactive power management was studied in relation to different load power factors and different degrees of PV penetration. Furthermore, the study focused on the topologies of the inverters and their implementation in the renewable energy grid.

\section{REFERENCES}

[1] Ooi H. P. Gabriel, A.I. Maswood,“ NPC Photovoltaic Grid-Connected Inverter using Proportional-Resonant Controller" DOI: 10.1109/APPEEC. 2014.7066201 December 2014

[2] J. Pou ,GeorgiosKonstantinou "Low-voltage ridethrough capability of full-row connected cascaded $\mathrm{H}$ bridge converters" DOI: 10.1109/TENCON. 2016.7848152 November 2016

[3] A. Camacho ,Miguel Castilla "Reactive Power Control for Distributed Generation Power Plants to Comply With
Voltage Limits During Grid Faults" DOI: 10.1109/TPEL.2014.2301463 November 2014

[4] JundiJia, Guangya Yang "A Review on Grid-Connected Converter Control for Short-Circuit Power Provision under Grid Unbalanced Fault" DOI: 10.1109/TPWRD.2017.2682164 March 2017

[5] L. B. G. Campanhol, S. A. O. da Silva, A. A. de Oliveira, and V. D. Bacon, "Dynamic performance improvement of a grid-tied pv system using a feedforward control loop acting on the npc inverter currents," IEEE Transactions on Industrial Electronics, vol. 64, no. 3, pp. 2092-2101, 2017.

[6] M. Schaefer, W. Goetze, M. Hofmann, F. Bayer, D. Montesinos-Miracle, and A. Ackva, "Direct current control for grid-connected diode-clamped inverters," IEEE Transactions on Industrial Electronics, vol. 64, no. 4,pp. 3067-3074, 2017.

[7] S. Kouro, J. I. Leon, D. Vinnikov, and L. G. Franquelo, "Grid-connected photovoltaic systems: An overview of recent research and emerging pv converter technology," IEEE Industrial Electronics Magazine, vol. 9, no. 1, pp. 47-61, 2015. 